\title{
28 Research Square \\ Comparative inhibition analysis of wild type and G671S catalytic site mutant of the SARS-CoV-2 RNA-dependent RNA polymerase
}

\section{Faisal Khan}

HEJ Research Institute of Chemistry, International Center for Chemical and Biological Sciences, University of Karachi, Pakistan

\section{Zarrin Basharat ( $\square$ zarrin.iiui@gmail.com )}

Jamil-ur-Rahman Center for Genome Research, Dr. Panjwani Center for Molecular Medicine and Drug Research, International Center for Chemical and Biological Sciences, University of Karachi, Karachi-75270, Pakistan.

\section{Research Article}

Keywords: SARS-CoV-2, Covid-19, Simulation, Screening, MM/PBSA, G671S, Remdesivir

Posted Date: December 28th, 2021

DOI: https://doi.org/10.21203/rs.3.rs-1203984/v1

License: (c) (i) This work is licensed under a Creative Commons Attribution 4.0 International License. Read Full License 


\section{Abstract}

Severe acute respiratory syndrome coronavirus 2(SARS-CoV-2) has wrecked a global havoc, after its emergence in Wuhan, Hubei province of China. It is known to have a distressing effect on the respiratory tract and has a high mortality rate. Till to date $(25$ Dec 2021$)$, total number of cases $(279,362,428)$ have been reported across the globe. Variable mutations have been reported on various geographical levels. In this study, we have analyzed G671S mutation of RdRp of the SARS-CoV-2, which has been reported in various strains globally, but importantly delta variant cases of Pakistan and has a serious impact on the protein structure. To study the conformational impact, we screened a Tibetan medicinal compound/Sowa Rigpa library against RdRp and compared the best docked compound (Kaempferol 3-0-gentiobioside) to the wild type and mutant RdRp against Remdesivir. A short simulation was used to validate the findings. Both remdesivir and our screened compound showed better inhibition for mutant, compared to the wild type RdRp.

\section{Introduction}

In December, 2019, an unknown viral outbreak, with pneumonia-like symptoms was reported in China (Wuhan, Hubei Province). It caused severe respiratory distress and was fatal in many cases. This deadly zoonotic virus led the World Health Organization (WHO) to declare a pandemic and lockdown was implemented in various parts of the world to alleviate spread (Rangayasami et al., 2021). Since the pandemic began, millions of cases have been reported, all over the world. Demographic studies estimated that approximately 100,871 people have been affected in China, 52,986,307 in America, and 1,293,081 in Pakistan till todate (25 December, 2021)[1].

SARS-CoV-2 is an RNA based viral strain, belonging to the subfamily ortho-coronavirinae, family coronaviridae and order Nidovirales (Fan et al., 2019). Its genomic sequence has $79.6 \%$ similarity with SARS-CoV and is $96 \%$ homologous to the bat corona virus RaTG13 (Zhou et al., 2020). SARS-CoV-2 has mutated over time span and altered its genomic structure. This variation impacts its spread and severity in different regions of the world (Dong et al., 2020). It follows lysogenic cycle based on RNA dependent RNA polymerase (RdRp). RdRp is composed of ORF1a, ORF1b, nsp7, nsp8 and nsp12 (a catalytic subunit), involved in virion replication and transcription (Gao et al., 2020). Recently, RdRp G671S (G15451A) mutant was observed in the delta variant strains. This variant has occurred $3,438,537$ times $(56.68 \%$ of all samples with NSP12 sequence) in 183 countries. The first strain with this amino acid change, collected in March 2020, was hCoV19/Germany/BW-FR0050/2020. Kannan et al. (2021) have reported a 100\% frequency of this mutation in Delta and Delta plus variant. Highest frequency (67.8\%) of this mutant in RdRp of Delta variant was reported by Fan et al. (2021). (Fig. 1).

Mutation based irregularity or dysfunction in viral machinery may lead to strengthen the efficacy of infection. Apart from phylogenetic and other sequence analysis, present study aimed to investigate whether this mutation influenced the binding of drugs to the mutant RdRp, compared to the wild-type control. In this study, bioinformatics methods like molecular docking, dynamics simulation and MM/PBSA energy analysis were used to analyze the impact.

[1] https://www.worldometers.info/coronavirus/

\section{Material And Methods}

In the present study, different bioinformatics methods and tools were used to find the efficacious drug for the inhibition of the RdRp (PDB ID: 7BV2), and hence, slowdown the replicating machinery. For thermodynamics and structural analyses, the 3-D homologous model for RdRp was retrieved from RCSB database explicitly the PDB-BLAST (PDB ID=7BV2) (Yin et al., 2020). Reference structure was mutated via PYMOL and both reference and mutated structure (G671S). 


\subsection{Molecular docking}

Screening of Tibetan traditional medicinal compound library (Basharat and Khoso, 2021) was done through structure based docking in MOE v 2016, and compared to the previously reported inhibitor, remdesivir (Elfiky, 2020; Yin et al., 2020; Kokic et al., 2021). The binding of molecules was priortized on basis of S-score, with a lower score having high binding affinity.

The descriptions for binding parameters are: placement=Triangle Matcher; Rescoring 01= London dG; refinement= forcefield, rescoring 02=affinity dG. A manual simulation input data was initiated and Molecular Mechanics PoissonBoltzmann surface Area (MM/PBSA) negative energy values were measured (Basharat et al., 2021). For analyzing ligand-receptor complex stability, the docking score for RdRp-remdesivir, RdRp-kaempferol 3-0-gentiobioside, G671Sremdesivir and G671S-kaempferol 3-0-gentiobioside was obtained.

\subsection{Energy changes}

Electrostatic Energy (ELE), Van der Waals contribution (VDW), Total Gas Phase energy (GAS), Non-polar and Polar

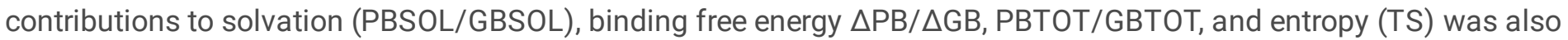
calculated $(\mathrm{kcal} / \mathrm{mol})$. The binding free energy $(\Delta \mathrm{Gbind})$ was calculated based on binding energy $(\Delta$ Ebind), solvation entropy (-T $\Delta \mathrm{Ssol})$ and conformational entropy (-T $\Delta \mathrm{Sconf})$. The enthalpy and the entropy were calculated by the MM/PB(GB)SA method (Wang et al., 2018) and empirical method, respectively. In order to gain a detailed profile of protein-ligand interactions and the contribution of each residue, the binding energy was decomposed per residue by using the decomposition module of the AMBER16 program.

\subsection{Dynamics simulation}

Top- scoring docking compounds were subjected to dynamics simulation for finding enhanced complex stability and identify interactions among them. High performance molecular dynamic simulation software Desmond from Schrodinger LLC was used to analyze dynamics, with OPLS3e force-field to resolve geometries. This was followed by energy minimization. The parameters in Desmond were: water solvation model, TIP3P; boundary conditions: orthorhombic box shape; size of box with a buffer donor/acceptor $(\mathrm{H}$ - bond distances distance $=2.5 \AA ̊$ each $)$. This was neutralized by adding $\mathrm{Na}^{+}$ions and $0.15 \mathrm{M}$ salt with $\mathrm{Na}^{+} / \mathrm{Cl}$ ions. The runtime for simulation was $15 \mathrm{~ns}$ for each complex, keeping the recording interval of $50 \mathrm{ps}$ trajectory and an energy equivalent to 1.2. Ensemble class was NPT with a temperature of $300 \mathrm{~K}$ and a pressure of 1.01325 bar. Once the simulation was done, then interaction analysis was initiated and manually interpreted.

\section{Results And Discussion}

\subsection{Molecular docking and energy analysis}

In SARS-CoV-2, RNA dependent RNA polymerase (RdRp) enzyme plays a pivotal role in genetic procreation by replicating the strain and then transcribe it into a protein sequence (Gao et al., 2020). It is the topographic (fundamental surface) point for antiviral drug to stop or slow down the viral replication via remdesivir binding (Yin et al., 2020). We studied its binding with reference to Sowa Rigpa and mutant G671S RdRp. Ligand-receptor complex docking scores for RdRpremdesivir, RdRp-3-0-gentiobioside, G671S-remdesivir and G671S-kaempferol 3-0-gentiobioside were -6.9, -9.1, -7.3, and -7.3, respectively. These values show that RdRp-kaempferol 3-0-gentiobioside interaction was more stable than RdRpremdesivir. It is pertinent to mention that Kaempferol 3-0-gentiobioside occurs in Meconopsis horridula. Its anti-tumor activity has previously been noted (Fan et al., 2015). 
Mutant values were even lower for RdRp-ligand binding, hinting at a better drug binding with mutant than the native protein. $\mathrm{N}$-terminus and $\mathrm{H}$-bond interactions of wild type and mutant RdRp were considerably altered (Fig. 2A, C). Similar findings were observed for RdRp-kaempferol 3-O-gentiobioside interaction.

Native RdRp-remdesivir binding free energies ( $\triangle \mathrm{PB}-17.55, \triangle \mathrm{GB}-22.19$; Table 1 ) were higher than the mutated $\mathrm{RdRp}$ G671S protein complex ( $\triangle \mathrm{PB}$-21.74, $\triangle \mathrm{GB}$-28.93). This shows a more stable interaction of remdesivir with mutant RdRp. Similarly, mutated Kaempferol3-0-gentiobioside ( $\triangle \mathrm{PB}-7.27, \triangle \mathrm{GB}-10.69)$ binding free energy was lower than native RdRp-kaempferol 3-0-gentiobioside ( $\triangle \mathrm{PB}$-3.35, $\Delta \mathrm{GB}$-10.36).Mazhari et al. (2021) have reported that this mutation conferred flexibility to the protein and our analysis shows that mutant G671S is better inhibited by both remdesivir and Kaempferol 3-0-gentiobioside, compared to wild type.

Table 1

$\mathrm{MM} / \mathrm{PB}(\mathrm{GB}) \mathrm{SA}$ values of docked complexes. Electrostatic energy (ELE), van der Waals contribution (VDW), total gas phase energy (GAS), non-polar and polar contributions to solvation (PBSOL/GBSOL). Final estimated binding free energy (deltaPB/ deltaGB) calculated from the terms above (PBTOT/GBTOT) and entropy (TS) in $\mathrm{kcal} / \mathrm{mol}$.

\begin{tabular}{|c|c|c|c|c|c|c|c|c|c|c|}
\hline & ELE & VDW & GAS & GBSOL & PBSOL & РВТОТ & GBTOT & TS & deltaPB & delatGB \\
\hline $\begin{array}{l}\text { Native- } \\
\text { remdesivir }\end{array}$ & -10.27 & -44.27 & -54.54 & 18.03 & 22.67 & -31.87 & -36.51 & 14.32 & -17.55 & -22.19 \\
\hline $\begin{array}{l}\text { Native- } \\
\text { kaempferol3- } \\
\text { O- } \\
\text { gentiobioside }\end{array}$ & -32.10 & -57.32 & -89.42 & 53.26 & 60.26 & -29.15 & -36.16 & 25.80 & -3.35 & -10.36 \\
\hline $\begin{array}{l}\text { Mutant } \\
\text { G671S- } \\
\text { remdesivir }\end{array}$ & -9.92 & -58.79 & -68.71 & 21.15 & 28.34 & -40.37 & -47.56 & 18.63 & -21.74 & -28.93 \\
\hline $\begin{array}{l}\text { Mutant } \\
\text { G671S- } \\
\text { kaempferol3- } \\
\text { O- } \\
\text { gentiobioside }\end{array}$ & 47.06 & -32.24 & 14.81 & -37.12 & -33.70 & -18.89 & -22.31 & 11.62 & -7.27 & -10.69 \\
\hline
\end{tabular}

\subsection{Dynamics simulation}

G671S RdRp-remdesivir shows a more stable interaction as RMSD of native RdRp shows increased value $(>2.8 \AA)$ after 8 ns, whereas it stays within pan of $2.5 \AA$ for G671S RdRp, even after 14 ns (Fig. 3). Similarly for native RdRp binding with kaempferol 3-O-gentiobioside, RMSD values were $>3 \AA$, after $10 \mathrm{~ns}$, whereas they were less than or round about $2.8 \AA$ for G671S RdRp-kaempferol 3-0-gentiobioside complex. This depicts that mutant protein was better inhibited by studied compounds. Hydrogen bonding plays a crucial role in protein-ligand interaction. A varied pattern of hydrogen bonding, hydrophobic, and ionic interactions was also observed for studied complexes. Mutant protein made no ionic interactions, while hydrogen bonds were also decreased in mutant-ligand complex formation (Fig. 4).

We conclude that Kaempferol 3-O-gentiobioside has equal potential of inhibiting G671S mutant RdRp as remdesivir, while mutant has high affinity towards these inhibitors when compared to the wild type.

\section{Declarations}

Competing interests: The authors declare no competing interests.

\section{References}


1. Basharat, Z., \& Khoso, A. (2021). Yaws (Endemic Treponematoses) Drug Discovery from Phytochemicals: An Informatics Protocol for Drug Target Identification to Phytochemical Inhibitor Screening and Validation. Neglected Tropical Diseases and Phytochemicals in Drug Discovery, 397-415.

2. Basharat, Z., Jahanzaib, M., \& Rahman, N. (2021). Therapeutic target identification via differential genome analysis of antibiotic resistant Shigella sonnei and inhibitor evaluation against a selected drug target. Infection, Genetics and Evolution, 94, 105004.

3. Dong, E., Du, H., \& Gardner, L. (2020). An interactive web-based dashboard to track COVID-19 in real time. The Lancet infectious diseases, 20(5), 533-534.

4. Elfiky, A. A. (2020). Ribavirin, Remdesivir, Sofosbuvir, Galidesivir, and Tenofovir against SARS-CoV-2 RNA dependent RNA polymerase (RdRp): A molecular docking study. Life sciences, 253, 117592.

5. Fan, J., Wang, Y., Wang, X., Wang, P., Tang, W., Yuan, W., ... Liu, Q. (2015). The antitumor activity of Meconopsis horridula Hook, a traditional Tibetan medical plant, in murine leukemia L1210 cells. Cellular Physiology and Biochemistry, 37(3), 1055-1065.

6. Fan, Y., Zhao, K., Shi, Z. L., \& Zhou, P. (2019). Bat coronaviruses in China. Viruses, 11(3), 210.

7. Fan, L. Q., Hu, X. Y., Chen, Y. Y., Peng, X. L., Fu, Y. H., Zheng, J. M., ... He, J. S. (2021). Biological Significance of the Genomic Variation and Structural Dynamics of SARS-CoV-2 B. 1.617. Frontiers in Microbiology, 2837.

8. Gao, Y., Yan, L., Huang, Y., Liu, F., Zhao, Y., Cao, L., ... Rao, Z. (2020). Structure of the RNA-dependent RNA polymerase from COVID-19 virus. Science, 368(6492), 779-782.

9. Gao, Y., Yan, L., Huang, Y., Liu, F., Zhao, Y., Cao, L., ... Rao, Z. (2020). Structure of the RNA-dependent RNA polymerase from COVID-19 virus. Science, 368(6492), 779-782.

10. Kannan, S. R., Spratt, A. N., Cohen, A. R., Naqvi, S. H., Chand, H. S., Quinn, T. P., ... Singh, K. (2021). Evolutionary analysis of the Delta and Delta Plus variants of the SARS-CoV-2 viruses. Journal of autoimmunity, 124, 102715.

11. Kokic, G., Hillen, H. S., Tegunov, D., Dienemann, C., Seitz, F., Schmitzova, J., ... Cramer, P. (2021). Mechanism of SARSCoV-2 polymerase stalling by remdesivir. Nature communications, 12(1), 1-7.

12. Rangayasami, A., Kannan, K., Murugesan, S., Radhika, D., Sadasivuni, K. K., Reddy, K. R., \& Raghu, A. V. (2021). Influence of nanotechnology to combat against COVID-19 for global health emergency: A review. Sensors International, 100079.

13. Wang, C., Greene, D. A., Xiao, L., Qi, R., \& Luo, R. (2018). Recent developments and applications of the MMPBSA method. Frontiers in molecular biosciences, 4, 87

14. Yin, W., Mao, C., Luan, X., Shen, D. D., Shen, Q., Su, H., ... Xu, H. E. (2020). Structural basis for inhibition of the RNAdependent RNA polymerase from SARS-CoV-2 by RDV. Science, 368(6498), 1499-1504.

15. Yin, W., Mao, C., Luan, X., Shen, D. D., Shen, Q., Su, H., ... Xu, H. E. (2020). Structural basis for inhibition of the RNAdependent RNA polymerase from SARS-CoV-2 by RDV. Science, 368(6498), 1499-1504.

16. Zhou, P., Yang, X. L., Wang, X. G., Hu, B., Zhang, L., Zhang, W., ... Shi, Z. L. (2020). A pneumonia outbreak associated with a new coronavirus of probable bat origin. nature, 579(7798), 270-273.

\section{Figures}




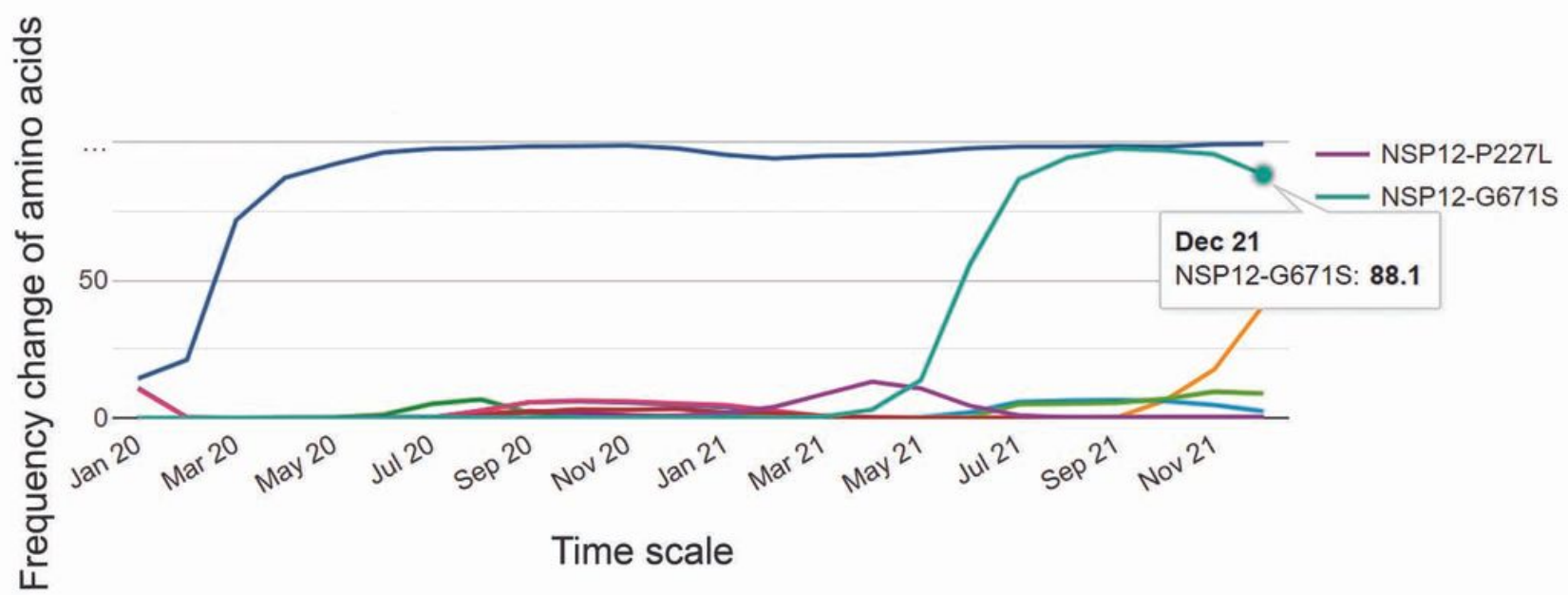

Figure 1

Average monthly frequency of amino acid changes in the RdRp protein. Mutants in $>2 \%$ of circulating strains are represented. Frequency of occurrence was 63\% in June 2021, 89\% in July 2021 and $~ 88 \%$ in December 2021, for G671S mutant. Source: https://mendel.bii.a-star.edu.sg/METHODS/corona/current/MUTATIONS/hCoV19_Human_2019_WuhanWIV04/hcov19_NSP12_mutations_table.html. 


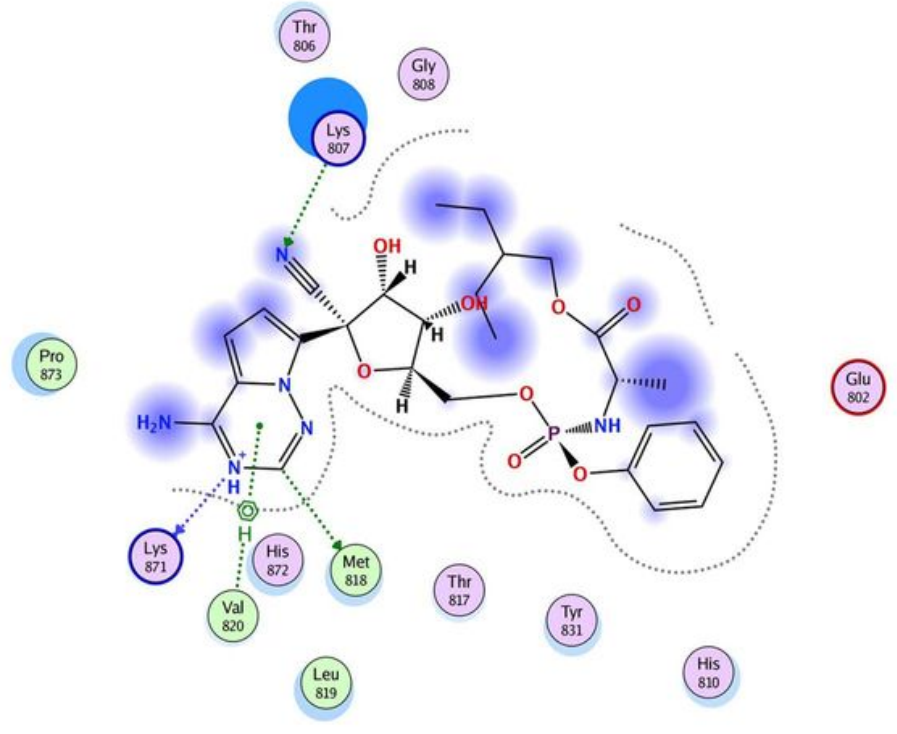

A

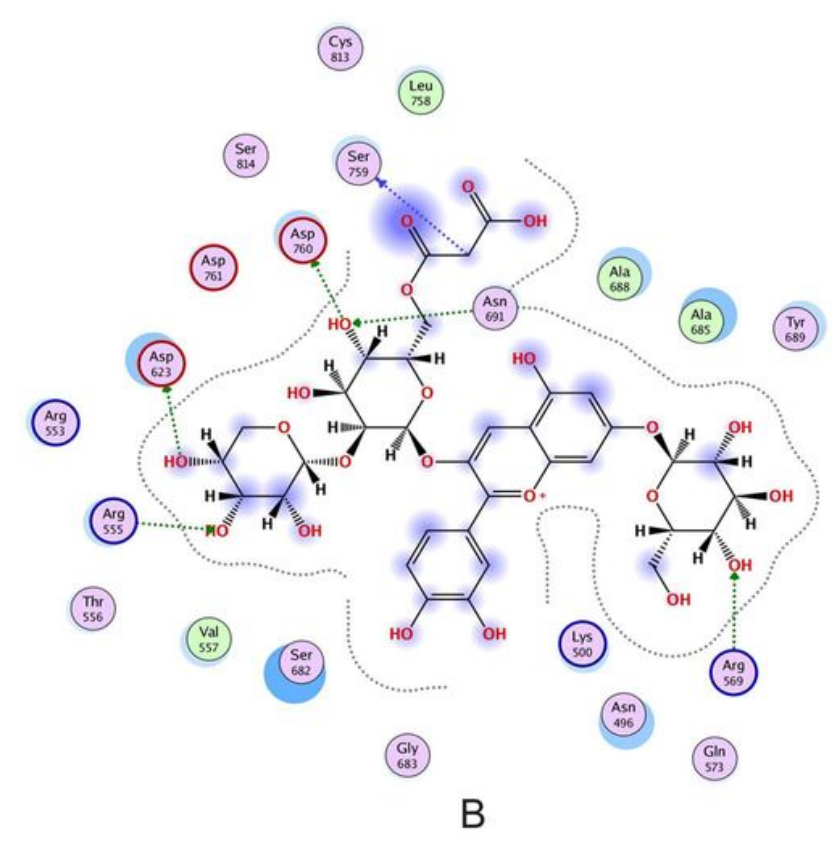

(4t5)

\section{Figure 2}

2D interaction of (A). RdRp-remdesivir (B). RdRp-Kaempferol 3-O-gentiobioside (C)G671S RdRp-remdesivir (D). G671S RdRp-kaempferol 3-O-gentiobioside interaction. 

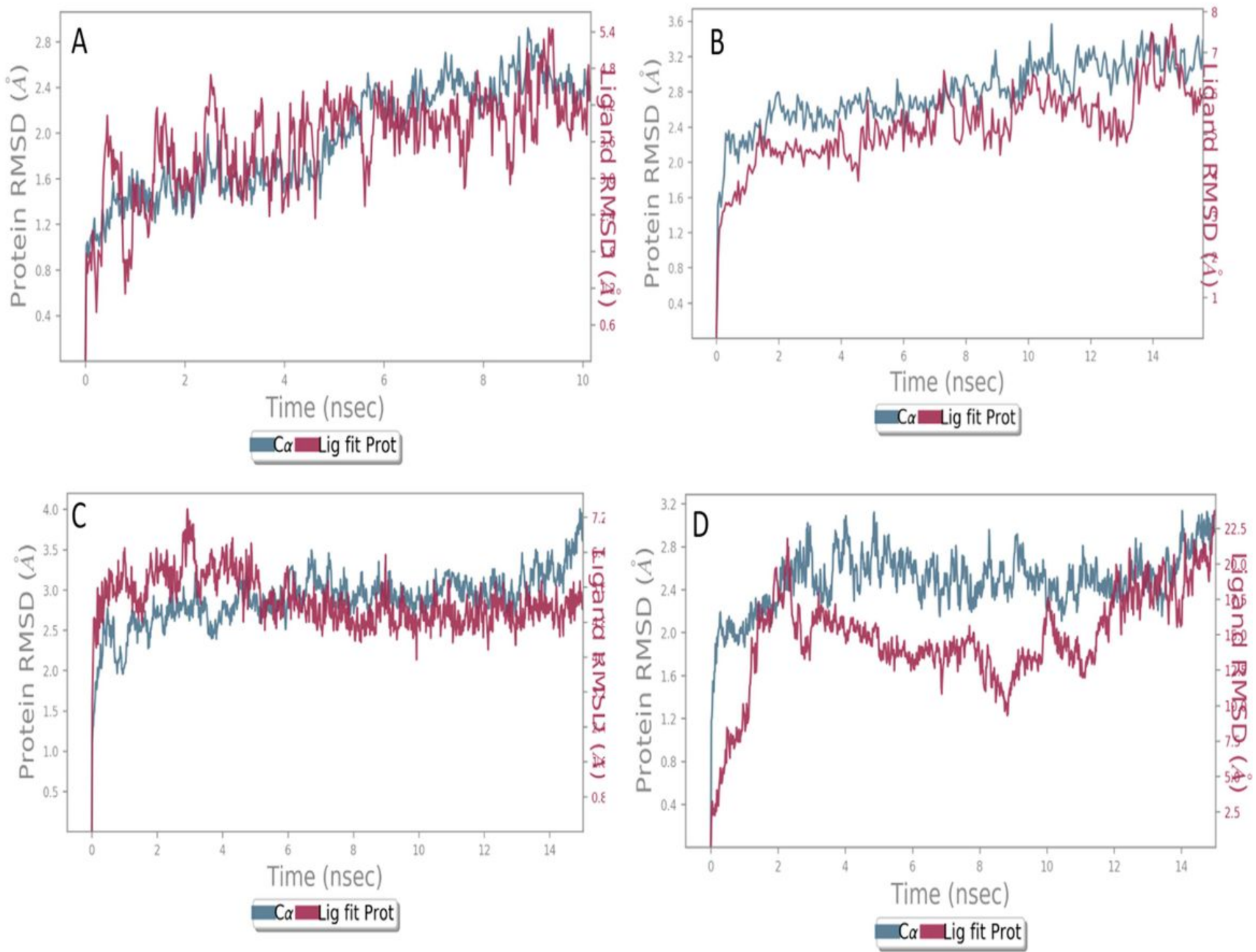

Figure 3

(A) RMSD of RdRp backbone interaction with remdesivir (B). Interaction of protein with kaempferol 3-0-gentiobioside (C). Interaction of G671S RdRp protein with remdesivir (D). Interaction of G671S mutated form of RdRp with kaempferol 3-0gentiobioside. 


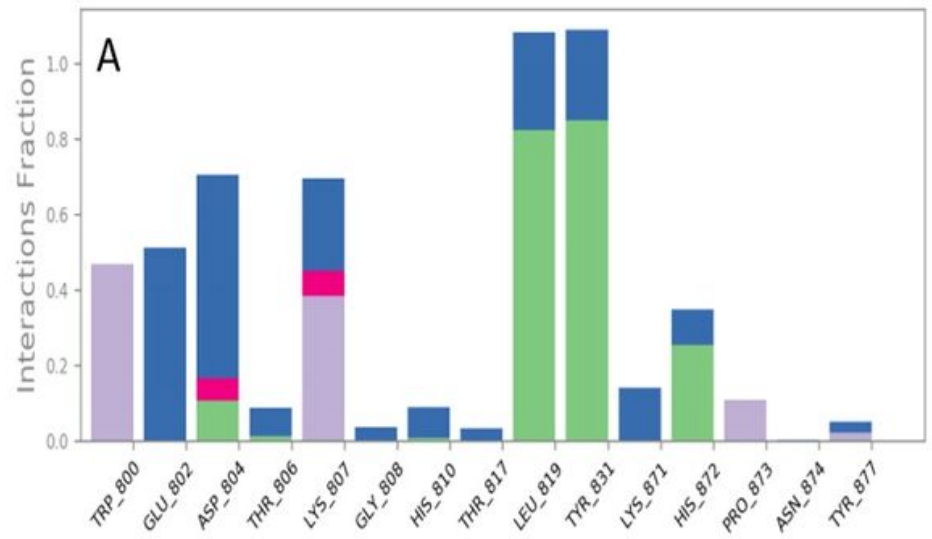

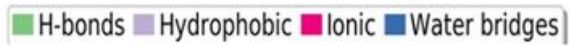

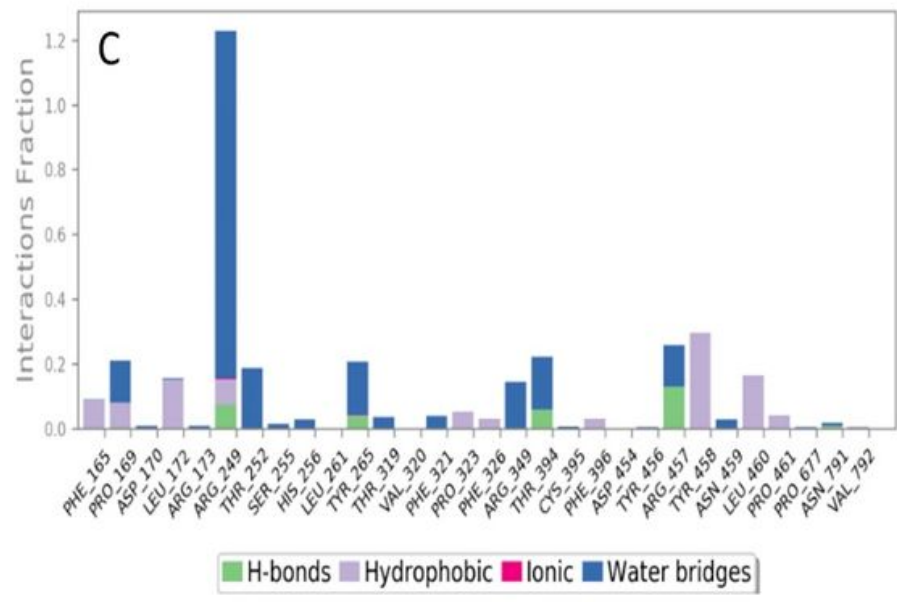

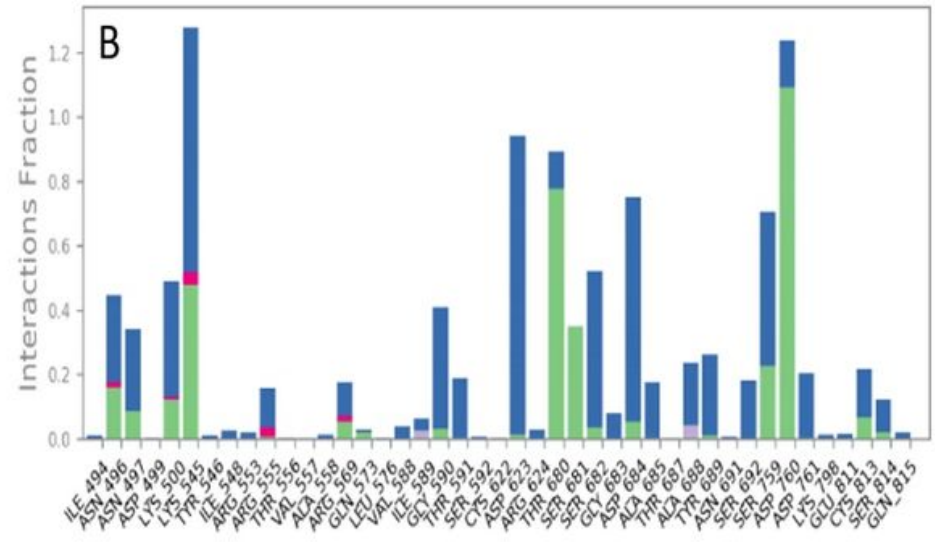

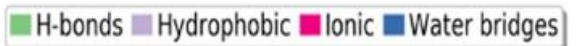

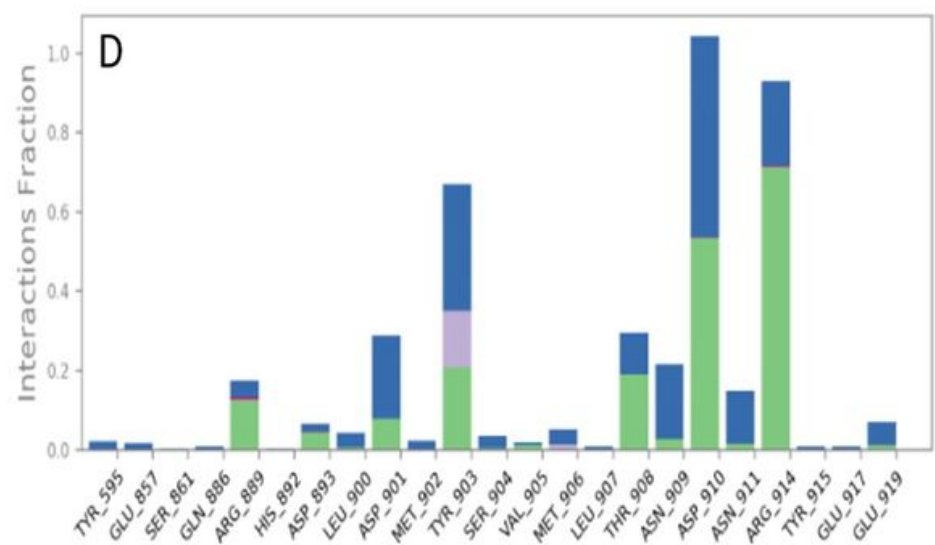

[ H-bonds $\mathbf{n}$ Hydrophobic $\square$ lonic $\square$ Water bridges

Figure 4

H-bonding fraction for (A) Native RdRp-remdesivir (B). Native RdRp-kaempferol 3-O-gentiobioside (C). G671 RdRpremdesivir (D) G671S RdRp and kaempferol 3-0-gentiobioside. 